\title{
Phytochemical Study of Adansonia digitata L Family Bombacaceae Cultivated in Egyp1
}

\author{
Nagwa. M. Ammar', Abdel-Monem M. Ateya ${ }^{2}$ and Asmaa A.Amer ${ }^{\prime}$ \\ ${ }^{1}$ Pharmacognosy Department, National Rescarch Center, Cairo, Egypt. \\ ${ }^{2}$ Pharmacognosy Dept., Faculty of Pharmacy, University of Zagazig, Egypt.
}

\section{ABSTRACT}

Phytochemical screening of the leaves of Adansonia digitata revealed the presence of flavonoids, tannins and sterols while the ripe fruit pulp was rich in carbohydrates, flavonoids and sterols. The polysaccharide content from the leaves $(20 \%)$ and ripe fruit pulp $(40 \%)$ were prepared, hydrolysed and analyzed qualitatively and quantitatively by GLC technique. Eleven sugars were identified in the mucilage hydrolysate of the leaves and nine sugars in the pectin hydrolysate of the pulp with rhamnose and glucose being the major, respectively. GLC analysis of the unsaponifiable matter as well as FAME of leaves, fruit pulp and seeds were conducted with significant differences. Also, analysis of the total proteins, total essential and non-essential amino acids content of the leaves, fruit pulp and seeds, were undertaken.

Key words: Adansonia digitata, Bombacaceae polysaccharide, lipoidal matter, proteins. essential, non essential amino acids

\section{INTRODUCTION}

Adansonia; A small genus of trees and shrubs, belongs to family Bombacaceae, comprising about 10 species of which some are indigenous to tropical Africa, Madagascar and Australia, including the will known African "Baobab" (Adansonia digitata L.) (Eggli, 2002). Adansonia digitata L.( Baobab) is one of the most important food plants, providing fruits, berries, nuts, seeds and leaves present in Ferlo (Diop et al., 2006). In addition, it has an exceedingly wide range of medicinal and traditional uses with numerous applications, including treatment of symptoms of infectious diseases (Gebauer et al., 2002). The fruit consists of (14 to 28\%) of pulp with a low moisture content, acidic, starchy, rich in vitamin $\mathrm{C}$, calcium and magnesium, so it is used as a traditional juice. The seeds are eaten fresh or dried and can also be roasted into a powder for use as a coffee substitute. The roasted seeds are very nutritious, rich in proteins and fats (Moctar et al., 2006).
Adansonia digitata $\mathrm{L}$. is the only species from this genus that has been grown in Egypt especially in Aswan. Due to its economic importance, the authors tried to acclimatize and cultivate the plant in the Agricultural Experimental Station of the National Research Center (NRC) at Nubareya, western Egypt. Conflicting reports were traced for African plants but nothing was reported about the chemistry and the biology of the Egyptian species. Therefore, the objective of this study was to investigate the phytochemical constituents of the Egyptian tree for instance, polysaccharides, lipoidal matter, protein and amino acids contents were undertaken for the leaves, fruit pulp and seeds. This is the first report about these constituents in this plant.

\section{MATERIALS and METHODS Plant material}

Samples of Adansonia digitata L. leaves and fruits were collected from Plant Island, Aswan, September 2009. The plant 
was kindly authenticated by $\mathrm{Dr}$. Mohammed flacicbaty, Department of Botany, National Rescarch Centre (NRC) and by Mrs. Tereez Labib, Consultant of Plant Taxonomy at the Monistry of Agriculture and Director of Drmen Betanical Garden, Giza, Egypt. The fruis were crushed to obtain powdered pulp and secds seeds and leaves were grinded into suitable size.

\section{Reference materials}

Reforence standards of carbohydrates, sterols, hydrocarbons, fatty acids and amino acids were obtained from the Department of Pharmacognosy, Faculty of Phamacy, Caim University

\section{Methods}

Phytochemical screening tests

The air-dried powdered leaves, fruit pulps and secds of Adansonia digitata L vere, scparately subjected to the following prehminary phyochemical screening tests according to the reported procedures. Carbohydrates and /or glycosides (Molisch. 1886). sterols and for triterpenes (Saliowski, 1872, Licbermann and Burchard, 1890), tannuns (Treose and Evans, 1078), (Babaa a d, 1981), flavonords, free and combined (Seike), 1902). anthraquinones, free and combined (Borntrager, 1880), (Walle, 1967), alkaloids and / or nirogenous compounds (Balbas of a.. 1981), saponins (Wall er al, 1954) and coumarus (Lepper, 1960).

Iurestigation of polysaccharide costent (Landlow, 1949. The Namonal Farmulam,
1975)

\footnotetext{
One hundred grams of powdered leaves and fina pulp were extracted
}

separately by cold extraction method (C.E.M.). Each extract was precipitated to obtain its polysaccharide content. Each precipitate was purified, submitted to gel formation test, hydrolysed and analyzed using GLC method using HP 6890.

\section{Investigation of lipoidal matter content}

The residue of the petroleum ether extract $(1.0 \mathrm{~g})$ for each of (leaf, fruit pulp and seeds) produced from the continuous extraction apparatus (Soxhlet apparatus) was saponified according to reported methods (Tsuda et al., 1960), (Finar, 1967).

Both the unsaponifiable and saponifiable fractions were extracted and analysed using GLC-MS (Agilent Technologies $6890 \mathrm{~N}$ (Networ GC system).

Investigation of total protein and total amino acid content ( $\mathrm{Ma}$ and Zuazage, 1942)

Determination of the total protein contents for leaves, fruit pulp and seeds was carried out by micro-Kjeldahl method using boric acid modification as described by $\mathrm{Ma}$ and Zuazage (1942) using Gerhardt distillation apparatus.

\section{Apparatus \\ GLC analysis of Polysaccharide contents \\ Using HP 6890) with the following conditions:}

Column: ZB-1701, $30 \mathrm{~m} \mathrm{X} 0.25 \mathrm{~m}$ X0.25 $\mu \mathrm{m}$, Stationary phase: $14 \%$ cyanopropyl phenyl methyl, Carrier gas: Helum (with Flow rate: $1.2 \mathrm{ml} / \mathrm{min}$, Pressure: $10.6 \mathrm{psi}$ and Velocity: $41 \mathrm{~cm} / \mathrm{sec}$ ), Injector chamber temperature: $250^{\circ} \mathrm{C}$, Back inlet with( Split ratio: 1 10. Split flow: 11.9 $\mathrm{ml} \mathrm{min}$. Total flow: $18.7 \mathrm{ml} / \mathrm{min}$, Gas saver flow: $120 \mathrm{ml}$ min and Saver time: $20 \mathrm{~min}$,) Oven with $\left(150^{\circ} \mathrm{c}\right.$ as Initial temp. , 2 min as 
imitial time, $7^{\circ} \mathrm{c} / \mathrm{min}$ rate and $200^{\circ} \mathrm{C}$ is a final temp. , 20 min.as a final time )and FID Detector (Temp $270{ }^{\circ} \mathrm{C}$, air flow 450 $\mathrm{ml} / \mathrm{min}$, and $\mathrm{H}_{2}$ flow $40 \mathrm{ml} / \mathrm{min}$ )

GLC analysin of the lipoidal matter contents

(Agilent Technologies $6890 \quad \mathrm{~N}$ Dvetwor GC system)) with the following conditions Unsaponifiable Matter: Column, capillary column of fused silica. $30 \mathrm{~m}$ lengh, $320 \mu \mathrm{m} \mathrm{D}$ and $0.25 \mu \mathrm{m}$ thichness; Stationary phase, HP-5 Phenyl Methl Siloxane: Carrier gas, Helium at 30 mi min. Nitogen at $30 \mathrm{ml} / \mathrm{min}$. Air $300 \mathrm{ml}$ min, Ramps, $8^{\circ} \mathrm{c}$ min Final temp $280^{\circ} \mathrm{c}$. Final time $20 \mathrm{~mm}$; Oven, Initial temp $70^{\circ} \mathrm{s}$ at intral time 1 min.: lnjector temperature. $280^{\circ} \mathrm{C}$; Detector Temp., $300^{\circ} \mathrm{C}$, Detector. FII)

Farty Acid Methyl Esters: Column. Capilary column of fused silica, $30 \mathrm{~m}$ lenghi, $320 \mu \mathrm{m} D$ and $0.25 \mu \mathrm{m}$ thuckness; Stanonary phase, MP-5 Phenyl Methy! Silexane; Comtier gas, Hehum at $30 \mathrm{ml}$ min, Nitogen al $30 \mathrm{mlmin}$, Air $300 \mathrm{ml} \mathrm{min}$, Ramp̧s, $8^{\circ} \mathrm{c} / \mathrm{min}$.

Final temp 200\%. Final tume 20 min; Over, Intial temp $70 \%$ at inual sume $2 \mathrm{~mm}$. Injector temperarare, $2500^{\circ} \mathrm{C}$; Detector Temp, 2806. Detector, FID.

A mine acis anahers (LC 300 amino acd analyer, Fppendorf-Germany) wah the following conduren Flow rate $0.2 \mathrm{ml}$ min. pressure of buffer form 0 to 50 bar, pressure of reagent from 0 - 150 bar and reaction temperature $123^{\circ} \mathrm{C}$

\section{RESULTS and DISCUSSION}

\section{Phytochemical screening}

Prelimmary phytochemical screening revealed that the dried powdered leaves, fruit pulp and seeds of Adansonia digitata $\mathrm{L}$. contuan, carbohydrates and/or glycosides, sterols and/or triterpenes, flavonoids (free and combened) and coumarins; while anthraguinones, alkaloids and saponins being absent, phile tannins was present only in leaves.

Investigation of the polysaccharide content

Precipitation and purification of polysaccharide content of the leaves and fruit pulp of Admsonia digitala $\mathrm{L}$. yielded mucilage $(20 \%)$ and pectin $(40 \%)$, respectively

GLC analysis of polysaccharide bydrolysate of leaves (Table 1) revealed the identification of eleven sugars with thamnose as the major one $(56.8 \%)$ followed by galactose $(10.3 \%)$. while, the hyrolysate of the fruil pulp (Table 2) rovealed the udentification of nine sugars with glucose as the major one $(532 \%$ \%) followed by arabinose $(21.2 \%)$ 
Table (1): GLC analysis of poly saccharide hydrolysate of leaves of Adansonia digilata L.

\begin{tabular}{llcc}
\hline Name & & Retention time & Relative area \% \\
\hline 1 & Arabinose & 7.545 & 11.476 \\
2 & Xylose & 7.562 & 3.341 \\
3 & Ribose & 7.817 & 0.902 \\
4 & Rhamnose & 8.355 & 56.801 \\
5 & Mannitol & 9.694 & 0.825 \\
6 & Sorbitol & 9.812 & 0.397 \\
7 & Fructose & 10.000 & 0.000 \\
8 & Galactose & 10.718 & 10.315 \\
9 & Mannose & 10.776 & 4.124 \\
10 & Glucose & 10.927 & 9.357 \\
11 & Galacturonic acid & 11.791 & 0.982 \\
12 & Glucuronic acid & 14.469 & 1.481 \\
\hline
\end{tabular}

$\frac{\text { Table (2): GLC analysis of polysaccharide hydrolysate of fruit pulp of Adansonia digitala } \mathrm{L}}{\text { Name }}$

\begin{tabular}{llcc}
\hline Name & & Retention time & Relative area \% \\
\hline 1 & Arabinose & 7.501 & 21.251 \\
2 & Xylose & 7.567 & 1.411 \\
3 & Ribose & 7.805 & 0.892 \\
4 & Rhamnose & 8.339 & 2.891 \\
5 & Mannitol & 9.716 & 0.000 \\
6 & Sorbitol & 9.809 & 4.355 \\
7 & Fructose & 10.000 & 0.000 \\
8 & Galactose & 10.721 & 3.425 \\
9 & Mannose & 10.780 & 2.135 \\
10 & Glucose & 10.977 & 53.278 \\
11 & Galacturonic acid & 11.816 & 10.324 \\
12 & Glucuronic acid & 14.469 & 0.000 \\
\hline
\end{tabular}

Investigation of the lipoidal matter content

\section{a-GLC analysis of lipoidal matter of Leaves}

GLC analysis of the unsaponifiable matter of leaves (Table 3 ) revealed the identification of 17 compounds representing $(73.91 \%)$ of the total compounds. The major compounds were n-tetracosane $\left(C_{24} \quad 15.05\right.$ $\%)$ and n-nonacosane $\left(\mathrm{C}_{29} \quad 10.17 \%\right)$. Four steroidal and one triterpenoidal compounds were identified representing $(23.53 \%)$ and $(5.88 \%)$ respectively. Stigmasterol $(9.77$ $\%)$ and $\beta$-sitosterol $(5.24 \%)$ are the major steroidal compounds.

\section{GLC analysis of FAME of leaves} (Table 4) resulting in the identification of 10 compounds representing $(90.90 \%)$ of the total matter. The major compounds were heptacosylic acid ( $\left.\mathrm{C}_{270} 40.62 \%\right)$ and palmitic acid $\left(\mathrm{C}_{160} 27.91 \%\right)$. Six saturated fatty acids represent $(60 \%)$ of the total identified compounds with heptacosylic acid $\left(\mathrm{C}_{270}\right)$ being the major one Four unsaturated fatty acid represent $(40 \%)$ of the total identified compounds with oleic acid $\left(\mathrm{C}_{18.1}\right)$ being the major one. Mono-, di-, triunsaturated fatty acids represent $(20 \%)$, $(10 \%)$ and $(10 \%)$ respectively. 
Table (3): GLC analysis of the unsaponifiable matter of leaves of Adansonia digitata $\mathrm{L}$.

\begin{tabular}{|c|c|c|c|c|}
\hline Peak No. & Name & Retention time & $\begin{array}{c}\text { * Relative Retention } \\
\text { Time }\left(\mathrm{RR}_{1}\right) \\
\end{array}$ & Area \% \\
\hline 1 & $n$ - hexadecane $\left(C_{10}\right)$ & 16.108 & 0.677 & 1.095 \\
\hline 2 & n- octadecane $\left(C_{18}\right)$ & 17.666 & 0.742 & 5.773 \\
\hline 3 & $n-$ nonacosane $\left(C_{10}\right)$ & 19.284 & 0.810 & 3.573 \\
\hline 4 & n- eicosane $\left(C_{20}\right)$ & 19.688 & 0.827 & 8.466 \\
\hline 5 & $\mathrm{n}$ - docosane $\left(\mathrm{C}_{22}\right)$ & 21.939 & 0.922 & 2.179 \\
\hline 6 & n-tetracosane $\quad\left(C_{24}\right)$ & 23.804 & 1 & 15.051 \\
\hline 7 & Unknown & 24.689 & 1.037 & 1.103 \\
\hline 8 & $n$-pentacosane $\left(C_{25}\right)$ & 25.042 & 1.052 & 1.546 \\
\hline 9 & $n$ - hexacosane $\left(C_{20}\right)$ & 26.257 & 1.103 & 8.381 \\
\hline 10 & $\mathrm{n}$ - heptacosane $\left(\mathrm{C}_{27}\right)$ & 26.855 & 1.128 & 2.604 \\
\hline 11 & Unknown & 27.294 & 1.147 & 2.893 \\
\hline 12 & $\mathrm{n}$-octacosane $\left(\mathrm{C}_{28}\right)$ & 27.959 & 1.175 & 4.063 \\
\hline 13 & n-nonacosane $\left(C_{29}\right)$ & 29.311 & 1.231 & 10.176 \\
\hline 14 & Unknown & 29.873 & 1.255 & 1.801 \\
\hline 15 & $n$-triacontane $\left(C_{30}\right)$ & 30.765 & 1.292 & 1.888 \\
\hline 16 & Cholesterol & 31.357 & 1.317 & 1.614 \\
\hline 17 & Campasterol & 31.963 & 1.343 & 1.230 \\
\hline 18 & Unknown & 32.852 & 1.380 & 4.630 \\
\hline 19 & Stigmasterol & 33.264 & 1.397 & 9.778 \\
\hline 20 & $\beta$ - sitosterol & 33.884 & 1.423 & 5.240 \\
\hline 21 & Unknown & 34.844 & 1.464 & 4.639 \\
\hline 22 & $\beta$-amyrin & 36.109 & 1.517 & 0.988 \\
\hline 23 & Unknown & 37.901 & 1.592 & 1.277 \\
\hline
\end{tabular}

* $\left(\mathrm{RR}_{4}\right)$ : Retention time relative to $\mathrm{n}$-tetracosane

Table (4): GLC analysis of FAME of Leaves of Adansonia digitata $L$.

\begin{tabular}{lcccc}
\hline Peak No. & Name & Retention time & $\begin{array}{c}\text { *Relative } \\
\text { Retention Time } \\
\left(\mathrm{RR}_{1}\right)\end{array}$ & Area \% \\
\hline 1 & Myristic acid $\left(\mathrm{C}_{14}\right)$ & 12.134 & 0.803 & 2.198 \\
2 & Palmitic acid $\left(\mathrm{C}_{160}\right)$ & 15.120 & 1 & 27.918 \\
3 & Palmatulonic acid $\left(\mathrm{C}_{161}\right)$ & 15.337 & 1.014 & 3.114 \\
4 & Margaric acid $\left(\mathrm{C}_{170}\right)$ & 16.928 & 1.120 & 3.976 \\
5 & Stearic acid $\left(\mathrm{C}_{180}\right)$ & 18.182 & 1.203 & 5.627 \\
6 & Oleic acid $\left(\mathrm{C}_{181}\right)$ & 18.657 & 1.234 & 4.492 \\
7 & Linoleic acid $\left(\mathrm{C}_{182}\right)$ & 20.173 & 1.334 & 2.935 \\
8 & Linolenic acid $\left(\mathrm{C}_{183}\right)$ & 21.173 & 1.400 & 3.429 \\
9 & Unknown & 24.455 & 1.617 & 3.725 \\
10 & Cerotic acid $\left(\mathrm{C}_{200}\right)$ & 30.872 & 2.042 & 1.954 \\
11 & Hentacosylic $A c i d\left(C_{270}\right)$ & 31.354 & 2.074 & 40.625 \\
\hline
\end{tabular}




\section{b- GLC analysis of lipoidal matter of fruit pulp}

GLC analysis of the unsaponifiable matter of fruit pulp (Table 5) revealed the identification of 25 compounds representing $(75.76 \%)$ of the total compounds. The major compounds were $\beta$-sitosterol (16.34337\%) and $n$ - Heneicosane $\left(C_{21}\right)(15.55765 \%)$. Four steroidal and two triterpenoidal compounds were identified representing $(16 \%)$ and $(8 \%)$ respectively. $\beta$-sitosterol $(16.34337 \%)$ was the major steroidal one.

GLC analysis of FAME of fruit pulp compounds representing $(83.33 \%)$ of the total compounds. The major compounds were palmitic acid $\left(\mathrm{C}_{160} 47.27 \%\right)$ and oleic acid $\left(C_{18}, 19.74 \%\right)$. Five saturated fatty acids represent $(50 \%)$ of the total identified compounds with palmitic acid $\left(\mathrm{C}_{16.0}\right)$ being the major one Five unsaturated fatty acid represent $(50 \%)$ of the total identified compounds with oleic acid $\left(C_{18}\right)$ being the major unsaturated one. Mono-, di-, tri- and poly- unsaturated fatty acids represent $(20 \%)$, $(10 \%),(10 \%)$ and $(10 \%)$ respectively.

(Table 6) resulting in the identification of 10

Table (5): GLC analysis of the unsaponifiable matter of fruit pulp of Adansonia digitata $\mathrm{L}$.

\begin{tabular}{|c|c|c|c|c|}
\hline Peak No. & Name & Retention lime & (RRT) & Area $\%$ \\
\hline 1 & $n$-Decane $\left(C_{10}\right)$ & 4.342 & 0.128 & 1.280 \\
\hline 2 & $n$-Undecane $\left(C_{11}\right)$ & 5.239 & 0.154 & 0.871 \\
\hline 3 & Unknown & 6.171 & 0.182 & 0.808 \\
\hline 4 & n-Dodecane & 6.746 & 0.199 & 1.534 \\
\hline 5 & n-Pentadecane $\left(C_{15}\right)$ & 11.671 & 0.345 & 4.834 \\
\hline 6 & Unknown & 15.050 & 0.458 & 0.736 \\
\hline 7 & $n$ - Hexadecane $\left(C_{16}\right)$ & 15.558 & 0.460 & 0.450 \\
\hline 8 & n- Heptadecane $\left(C_{17}\right)$ & 16.038 & 0.474 & 0.718 \\
\hline 9 & $\mathrm{n}$ - Octadecane $\left(\mathrm{C}_{18}\right)$ & 16.864 & 0.498 & 0.605 \\
\hline 10 & $n$ - Nonacosane $\left(C_{19}\right)$ & 17.838 & 0.527 & 11.118 \\
\hline 11 & n- Eicosane $\left(C_{20}\right)$ & 18.530 & 0.547 & 1.747 \\
\hline 12 & $\mathrm{n}$ - Heneicosane $\left(\mathrm{C}_{21}\right)$ & 19.864 & 0.587 & 15.557 \\
\hline 13 & Unknown & 20.792 & 0.615 & 0.959 \\
\hline 14 & $n$ - Docosane $\left(C_{22}\right)$ & 21.169 & 0.626 & 0.998 \\
\hline 15 & n-Tricosane $\left(C_{23}\right)$ & 21.930 & 0.648 & 3.560 \\
\hline 16 & n-Tetracosane $\quad\left(C_{24}\right)$ & 23.154 & 0.684 & 1.390 \\
\hline 17 & $n$-Pentacosane $\left(C_{2 s}\right)$ & 23.683 & 0.700 & 1.969 \\
\hline 18 & Unknown & 24.087 & 0.712 & 1.551 \\
\hline 19 & $\mathrm{n}$ - Hexacosane $\left(\mathrm{C}_{26}\right)$ & 24.999 & 0.739 & 2.019 \\
\hline 20 & Unknown & 25.867 & 0.764 & 3.663 \\
\hline 21 & $n$ - Heptacosane $\left(C_{27}\right)$ & 26.784 & 0.792 & 4.280 \\
\hline 22 & n-Octacosane $\left(C_{28}\right)$ & 27.849 & 0.823 & 0.988 \\
\hline 23 & Unknown & 28.228 & 0.834 & 1.552 \\
\hline 24 & $n$-Nonacosane $\left(C_{29}\right)$ & 29.017 & 0.858 & 3.659 \\
\hline 25 & $n$-Triacontane $\left(\mathrm{C}_{30}\right)$ & 29.608 & 0.875 & 1.594 \\
\hline 26 & Cholesterol & 29.899 & 0.884 & 2.819 \\
\hline 27 & Campasterol & 31.682 & 0.936 & 2.631 \\
\hline 28 & Stigmasterol & 32.329 & 0.956 & 3.129 \\
\hline 29 & $\beta=$ sitosterol & 33.823 & 1 & 16.343 \\
\hline 30 & Unknown & 34.437 & 1.018 & 2.927 \\
\hline 31 & Unknown & 35.353 & 1.045 & 2.819 \\
\hline 32 & $\beta$-amyrin & 36.144 & 1.068 & 0.555 \\
\hline 33 & a-amyrin & 36.866 & 1.089 & 0.323 \\
\hline
\end{tabular}


Table (6): GL.C analysis of FAME of fruit pulp of Adansonia digitata $\mathrm{L}$

\begin{tabular}{lcccc}
\hline Peak No. & Name & Retention time & $\begin{array}{c}\text { Relative Retcrtion } \\
\text { time }\left(R_{1}\right)\end{array}$ & Area \% \\
\hline 1 & Lauric acid $\left(\mathrm{C}_{12}\right)$ & 10.737 & 0.7172 & 1.710 \\
2 & Myristic acid $\left(\mathrm{C}_{14}\right)$ & 12.192 & 0.8144 & 4.004 \\
3 & Palmitic acid $\left(\mathrm{C}_{160}\right)$ & 14.970 & 1 & 47.279 \\
4 & Palmatulonic acid $\left(\mathrm{C}_{101}\right)$ & 16.402 & 1.0956 & 1.336 \\
5 & Margaric acid $\left(\mathrm{C}_{170}\right)$ & 16.991 & 1.1350 & 1.383 \\
6 & Stearic acid $\left(\mathrm{C}_{180}\right)$ & 17.899 & 1.1956 & 6.325 \\
7 & Oleic acid $\left(\mathrm{C}_{18}\right)$ & 18.492 & 1.2350 & 19.745 \\
8 & Linoleic acid $\left(\mathrm{C}_{182}\right)$ & 19.541 & 1.3050 & 6.459 \\
9 & Unknown & 19.932 & 1.3314 & 1.231 \\
10 & Linolenic acid $\left(\mathrm{C}_{183}\right)$ & 20.810 & 1.3907 & 5.471 \\
11 & Unknown & 21.724 & 1.451 & 1.213 \\
12 & Arachidonic acid $\left(\mathrm{C}_{204}\right)$ & 23.579 & 1.575 & 3.838 \\
\hline
\end{tabular}

* $\mathrm{RR}_{1}$ : Retention time relative to Palmitic acid $=1$

c- GLC analysis of lipoidal matter of seeds:

GLC analysis of the unsaponifiable matter of seeds (Table 7) revealed the identification of 21 compounds representing $(70 \%)$ of the total matter. The major compound was n-tetracosane $\left(\mathrm{C}_{24}\right.$ $60.455 \%)$. Three steroidal and two triterpenoidal compounds were identified representing $(14.29 \%)$ and $(9.5 \%)$ respectively of the total identified compounds. Stigmasterol $(1.56 \%)$ was the major sterol.

GLC analysis of FAME of seeds (Table 8) resulting in the identification of 10 compounds representing $(83.33 \%$ ) of the total matter. The major compounds were palmitic acid $\left(\mathrm{C}_{16.0} 38.54 \%\right)$ and oleic acid $\left(\mathrm{C}_{18.1} 33.28 \%\right.$ ). Five saturated Fatty acids represent $(50 \%)$ of the total identified compounds, palmitic acid $\left(\mathrm{C}_{160} 38.54 \%\right)$ and stearic acids $\left(\mathrm{C}_{18} 07.06 \%\right)$ are the major. While, five unsaturated fatty acid represent $(50 \%)$ of the total identified compounds. Mono-, di-, tri- unsaturated fatty acids represent $(20 \%),(10 \%),(10 \%)$ and $(10 \%)$, respectively. Oleic acid $\left(\mathrm{C}_{18}, 33.28 \%\right)$ and linoleic acid $\left(\mathrm{C}_{18} 24.32 \%\right)$ are the major. 
Table (7): GLC analysis of the unsaponifiable matter of seeds of Adansonia digitata $\mathrm{L}$.

\begin{tabular}{|c|c|c|c|c|}
\hline Peak No. & Name & Retention time & $\begin{array}{l}\text { Relative Retention } \\
\text { Time (RRT) }\end{array}$ & Area $\%$ \\
\hline 1 & Unknown & 6.800 & 0.282 & 0.225 \\
\hline 2 & n-Pentadecane $\left(C_{15}\right)$ & 14.236 & 0.591 & 0.439 \\
\hline 3 & Unknown & 15.071 & 0.626 & 0.767 \\
\hline 4 & n- Hexadecane $\left(\mathrm{C}_{16}\right)$ & 15.629 & 0.649 & 2.069 \\
\hline 5 & n- Heptadecane $\left(C_{17}\right)$ & 16.769 & 0.696 & 1.013 \\
\hline 6 & n- Octadecane $\left(\mathrm{C}_{18}\right)$ & 17.692 & 0.734 & 4.045 \\
\hline 7 & n- Nonacosane $\left(C_{19}\right)$ & 18.802 & 0.780 & 3.092 \\
\hline 8 & n- Eicosane $\left(C_{20}\right)$ & 19.756 & 0.820 & 8.303 \\
\hline 9 & $\mathrm{n}$ - Heneicosane $\left(C_{21}\right)$ & 20.826 & 0.864 & 1.597 \\
\hline 10 & Unknown & 21.249 & 0.882 & 1.411 \\
\hline 11 & n- Docosane $\left(C_{22}\right)$ & 22.014 & 0.914 & 2.543 \\
\hline 12 & n- Tricosane $\left(C_{23}\right)$ & 23.309 & 0.967 & 1.057 \\
\hline 13 & $n$-Tetracosane $\quad\left(C_{24}\right)$ & 24.094 & 1 & 60.455 \\
\hline 14 & $n$-Pentacosane $\left(C_{2 s}\right)$ & 25.086 & 1.041 & 3.135 \\
\hline 15 & n- Hexacosane $\left(C_{26}\right)$ & 26.249 & 1.089 & 1.004 \\
\hline 16 & n- Heptacosane $\left(C_{27}\right)$ & 26.859 & 1.115 & 0.539 \\
\hline 17 & Unknown & 27.208 & 1.131 & 0.978 \\
\hline 18 & Unknown & 27.928 & 1.159 & 0.433 \\
\hline 19 & $n$-Octacosane $\left(C_{28}\right)$ & 28.307 & 1.175 & 0.541 \\
\hline 20 & n-Nonacosane $\left(C_{29}\right)$ & 29.208 & 1.212 & 1.122 \\
\hline 21 & Unknown & 29.665 & 1.231 & 0.777 \\
\hline 22 & $n-T$ riacontane $\left(\mathrm{C}_{30}\right)$ & 30.702 & 1.274 & 0.398 \\
\hline 23 & Unknown & 31.347 & 1.301 & 0.577 \\
\hline 24 & Campasterol & 31.932 & 1.325 & 0.305 \\
\hline 25 & Unknown & 32.724 & 1.358 & 0.448 \\
\hline 26 & Stigmasterol & 33.118 & 1.375 & 1.560 \\
\hline 27 & Unknown & 33.768 & 1.402 & 0.360 \\
\hline 28 & $\beta$ - sitosterol & 34.723 & 1.441 & 0.418 \\
\hline 29 & $\beta$-amyrin & 36.093 & 1.498 & 0.210 \\
\hline 30 & $\alpha$-amyrin & 36.793 & 1.527 & 0.166 \\
\hline
\end{tabular}

- $\left(R_{1}\right)$ : Retention lime relative to n-Tetracosane 
Table (8): GLC analysis of FAME of seeds of Adansonia digitata $\mathrm{L}$

\begin{tabular}{|c|c|c|c|c|}
\hline Peak No. & Name & Retention time & $\begin{array}{l}\text { "Relative Retention } \\
\text { time }\left(\mathrm{RR}_{\mathrm{l}}\right)\end{array}$ & Area $\%$ \\
\hline 1 & Lauric acid $\left(\mathrm{C}_{12}\right)$ & 10.737 & 0.717 & 1.710 \\
\hline 2 & Myristic acid $\left(\mathrm{C}_{14}\right)$ & 12.192 & 0.814 & 4.004 \\
\hline 3 & Palmitic acid $\left(\mathrm{C}_{160}\right)$ & 14.970 & 1 & 47.279 \\
\hline 4 & Palmatulonic acid( $\left.\mathrm{C}_{16.1}\right)$ & 16.402 & 1.095 & 1.336 \\
\hline 5 & Margaric acid $\left(C_{170}\right)$ & 16.991 & 1.135 & 1.383 \\
\hline 6 & Stearic acid $\left(C_{180}\right)$ & 17.899 & 1.195 & 6.325 \\
\hline 7 & Oleic acid $\left(\mathrm{C}_{181}\right)$ & 18.492 & 1.235 & 19.745 \\
\hline 8 & Linoleic acid $\left(C_{182}\right)$ & 19.541 & 1.305 & 6.459 \\
\hline 9 & Unknown & 19.932 & 1.331 & 1.231 \\
\hline 10 & Linolenic acid $\left(\mathrm{C}_{18}{ }_{3}\right)$ & 20.810 & 1.390 & 5.471 \\
\hline 11 & Unknown & 21.724 & 1.451 & 1.213 \\
\hline 12 & Arachidonic acid $\left(\mathrm{C}_{20 \cdot 4}\right)$ & 23.579 & 1.575 & 3.838 \\
\hline
\end{tabular}

* $\mathrm{RR}_{1}$ : Retention time relative to Palmitic acid $=1$

Investigation of total protein and total amino acid content

The percentage of total protein in leaves, fruit pulp and seeds were found to be $9.625 \%, 2.125 \%$ and $12.688 \%$, respectively. Analysis of total amino acids content revealed the identification of $(7,5$ and 6$)$ essential amino acids and $(8,7$ and 8$)$ nonessential amino acids for leaves, fruit pulp and seeds, respectively. Both leucine and phenylalanine were the major essential amino acids in leaves and seeds while leucine and threonine were the major in the fruit pulp. Alanine and glutamic acid were the major non-essential amino acids in the leaves while glutamic acid and alanine were major in both seeds and fruit pulp. Essential amino acids methionine was present only in leaves and absent in both pulp and seeds. In contrast histidine was absent only in pulp. Non-essential amino acid, tyrosine was found in both leaves and seeds and absent in fruit pulp. 
V')l. 22, Issue. 1, pp, 26-38

Table (9) Total amino acids content of leaves of Adansonia digitata L..

\begin{tabular}{llcc}
\hline Essential amino acids: & Retention time & Relative area \% \\
\hline I & Threonine & 14.03 & 4.654 \\
2 & Methionine & 35.22 & 0.824 \\
3 & Isoleucine & 37.13 & 3.780 \\
4 & Leucine & 38.18 & 10.125 \\
5 & Phenylalanine & 42.80 & 5.682 \\
6 & Histidine & 50.23 & 2.142 \\
7 & Lysine & 53.60 & 3.009 \\
Non essential amino acids: & & \\
1 & Aspartic acid & 10.83 & 11.229 \\
2 & Serine & 15.28 & 7.115 \\
3 & Glutamic acid & 16.73 & 13.607 \\
4 & Glycine & 24.32 & 7.884 \\
5 & Alanine & 25.25 & 15.305 \\
6 & Cystine & 30.90 & 6.731 \\
7 & Tyrosine & 41.33 & 3.098 \\
8 & Arginine & 62.78 & 3.009 \\
\hline
\end{tabular}

Table (10): Total amino acids content of fruit pulp of Adansonia digitata

\begin{tabular}{llcc}
\hline Essential amino acids : & Retention time & Relative area \% \\
\hline 1 & Threonine & 13.73 & 4.953 \\
2 & Isoleucine & 36.92 & 3.111 \\
3 & Leucine & 37.98 & 10.255 \\
4 & Phenylalanine & 42.55 & 2.915 \\
5 & Lysine & 53.60 & 4.694 \\
Non essential amino acids : & & \\
1 & Aspartic acid & 10.53 & 12.928 \\
2 & Serine & 14.97 & 10.574 \\
3 & Glutamic acid & 16.28 & 19.611 \\
4 & Glycine & 23.97 & 8.912 \\
5 & Alanine & 24.88 & 16.528 \\
6 & Cystine & 30.50 & 3.374 \\
7 & Arginine & 61.78 & 2.135 \\
\hline
\end{tabular}


Table (11) Total amino acids content of sceds of Adansonia digitata $\mathrm{L}$.

\begin{tabular}{llcc}
\hline Essential amino acids & Retention time & Relative are3\% \\
\hline 1 & Threonine & 13.90 & 2.486 \\
2 & Isoleucine & 37,00 & 2.244 \\
3 & Leucine & 38,05 & 7.179 \\
4 & Phenylalanine & 42,63 & 4.166 \\
5 & Histidine & 50.13 & 1.954 \\
6 & Lysine & 53,47 & 3.973 \\
Non essential amino acids & & \\
1 & Aspartic acid & 10.72 & 8.206 \\
2 & Serine & 15.17 & 5.848 \\
3 & Glutamic acid & 16.53 & 20.942 \\
4 & Glycine & 24.17 & 9.887 \\
5 & Alanine & 5.07 & 19.841 \\
6 & Cystine & 30.72 & 4.988 \\
7 & Tyrosine & 41.20 & 1.630 \\
8 & Arginine & 61.78 & 6.661 \\
\hline
\end{tabular}

\section{REFERENCES}

1. The National Formulary, $14^{\text {th }}$ edition. (NF NIV) (1975). Washington: Official American Pharmaceutical Association. pp. 534.

2. Balbaa, S.L; Hilal, S.H.; and Zaki, A.Y. (1981). Medicinal Plants Constituents, $3^{\text {rd }}$ edition. Cairo: Dar El-Shaab Printing House. pp 366.

3. Borntrager, H.Z. (1880). Anal Chem. 19: 165, through Fairbairn, J.W. (1949). The active constituents of the vegetable purgative containing anthraquinones derivatives. J Pharm. 1: 683-694.

4. Diop, A.G., Sakho, M.; Dornier, M.; Cisse, M., and Reynes; M. (2006). The African baobab tree (Adansonia digilala L.): principal characteristics and uses, Fruits J. 61 (1): 55 - 69, C. A. 138 (26), 393437e, 2003

5. Eggli, Urs. (2002). Illustrated Handbook of Succulent Plants: Dicotyledons. Berlin Heidelberg New York: Springer-Verlag. pp.48.

6. Finar, I.L. (1967). Organic Chemistry, $5^{\text {th }}$ edition. London: Longmans Green and Co. Ltd. 1: 212.

7. Gebauer, J.; El-Siddig, K.; and Ebert, G. (2002). Baobab (Adansonia digitata L.): A Review on a Multipurpose Tree with Promising Future in the Sudan. Gartenbauwissenschaft. 67 (4): 155160, C. A. 138 (15), 218175a, 2003.

8. Laidlow, R.A.; and Percival, E.G.V. (1949). J Chem Soc. 2: 1603.

9. Lepper, H.A. (1960). Official methods of analysis of the association of official agriculture chemists, $9^{\text {th }}$ edition. Washington: Ass Offi Agri Chem.

10. Liebermann, $\mathrm{C}$. ; and Burchard, $H$. (1890). Chem Zent. 16: 1, through E! Deeb, K.S. (1985). Chromatographic and thermal analysis of certain volatile oil containing drugs. Ph.D. Thesis, Cairo: Cairo University. pp. 47.

11. Ma, T.S.; and Zauzage, C. (1942). Microkjeldahl determination of nitrogen, a new indicator and an improved rapid method. Industr Eng Chem Anal Ed. 14: 280-286.

12. Sacande, M.; Ronne, C.; Sanon, S.; and Joker, D. (2006). Seed leaflet Journal on line. 109.

13. Molisch, H. (1886). Monatsh Chem., 7, through Stank, J.; Gerny, M., Kocourek, J.; and Pacak, J., (1963). The Monosaccharides, Prague: Publishing 
House of Czechoslovak Academy of Science. pp. 872 .

14. Salkowski, F. (1872). Arch Ges Physiol. 6: 207, through; El Deeb, K.S.; (1985). Chromatographic and thermal analysis of certain volatile oil containing drugs. Ph.D. Thesis, Cairo: Cairo University.

15. Seikel. M.K. (1962).

Chromatographic methods of separation, isolation and identification of flavonoid compounds. In Geissman, T.A., editor. The Chemistry of Flavonoid Compounds. New York: Macmillan Co.pp. 34.

16. Trease, G.E.; and Evans, W.C. (1978). Text Book of Pharmacognosy, $8^{\text {th }}$ edition. London: Bailliere Tindall Cassel. pp. 536.

17. Tsuda, K.; Sakai, K.; Tanabe, K.; and Kishida, Y. (1960). Isolation of 22. dehydrocholestrol from Hypnea japonica. J Am Chem Soc. 82: 1442. 1443.

18. Wall, M.E.; Krieder, M.M.; Krewson, C.F.; Eddy, C.R.; William, J.J.; Carel, D.S. and Gentry, H.S. (1954). Steroidal saponin and other constituents. J Amer Pharm Assoc. 43: 1 .

19. Wallis, T.E. (1967). Text Book of Pharmacognosy, $5^{\text {th }}$ edition. London: J. and A. Churchill LTD. pp. 449. 


\section{لر امدة فيتوكيطانية على نبات ادانسونبا ليجيناتا المنزرع فى هصر

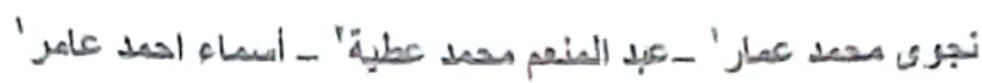

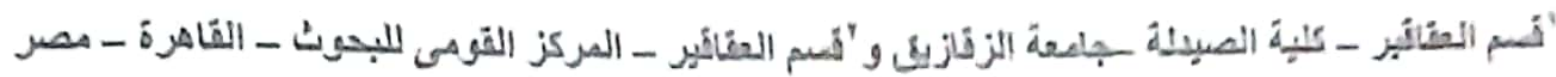

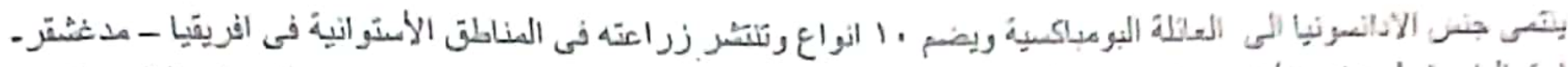

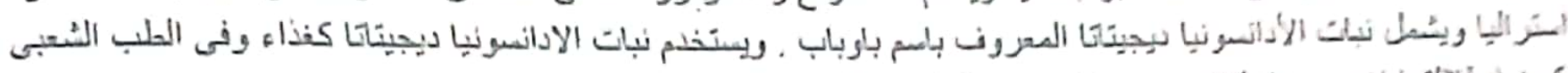

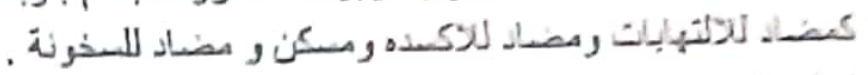

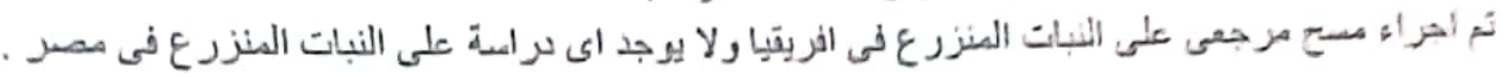

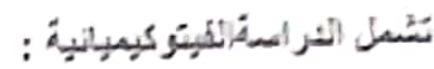

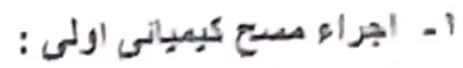

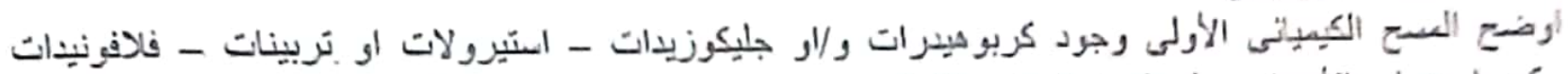

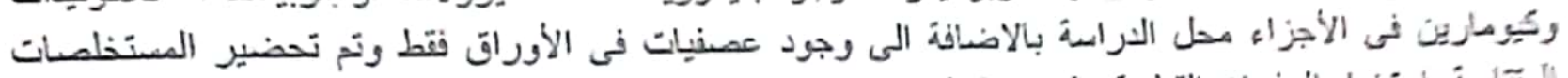

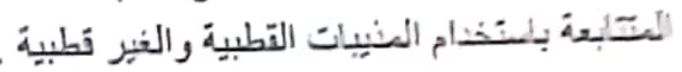

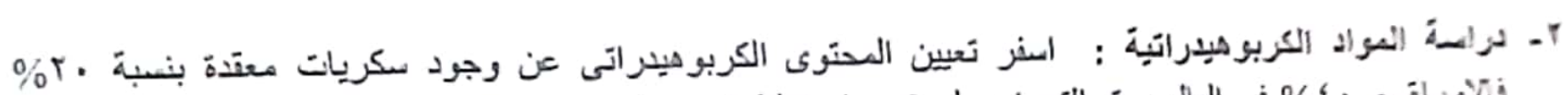

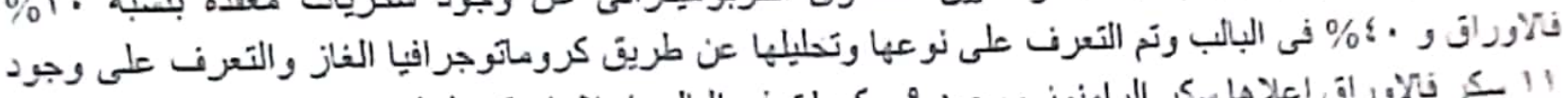

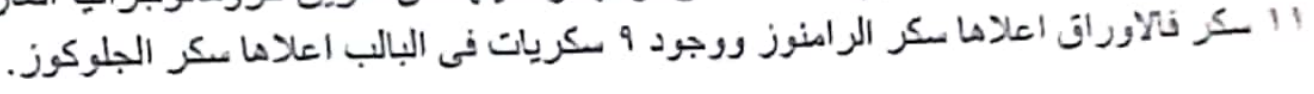

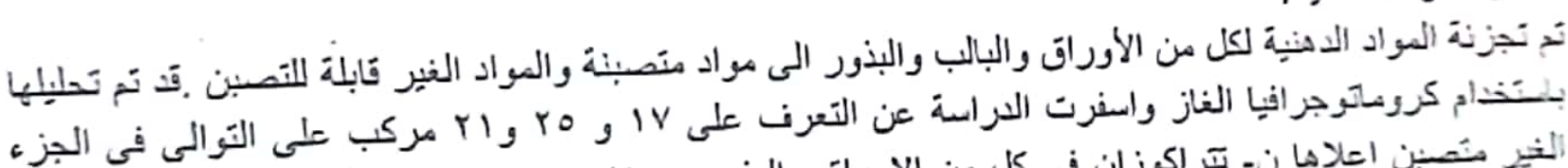

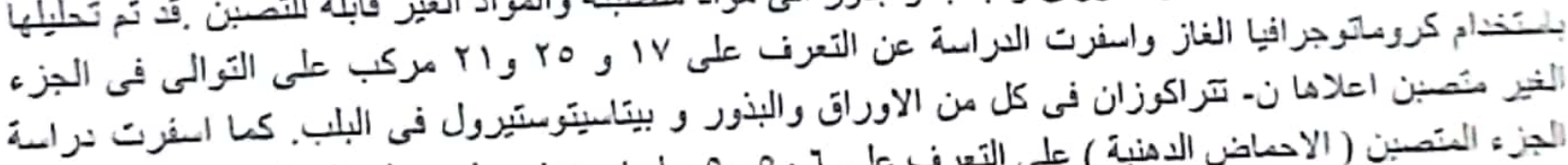

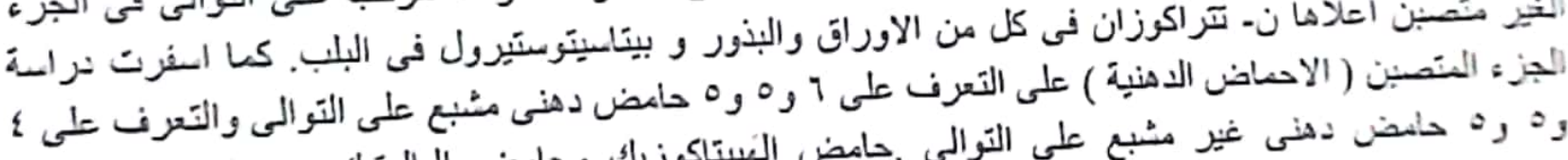

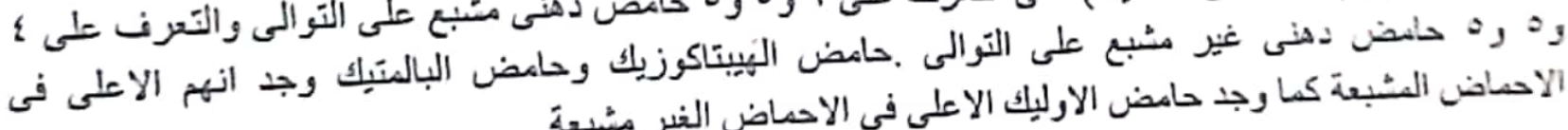

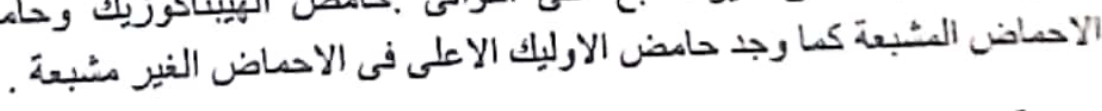

:- دراسة المتنوى البروتينى والأحماض الامينية.

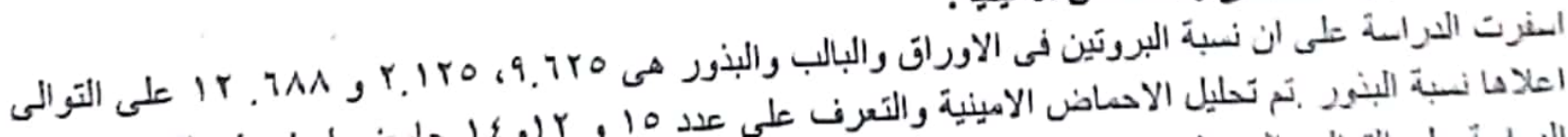

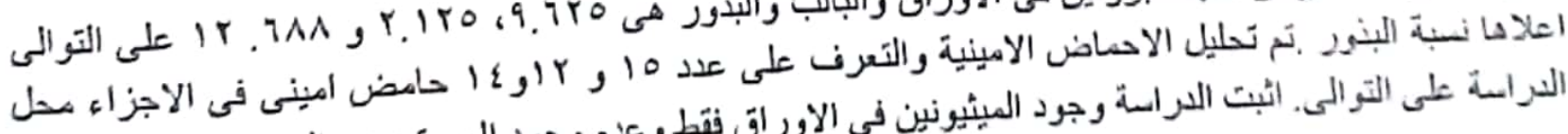

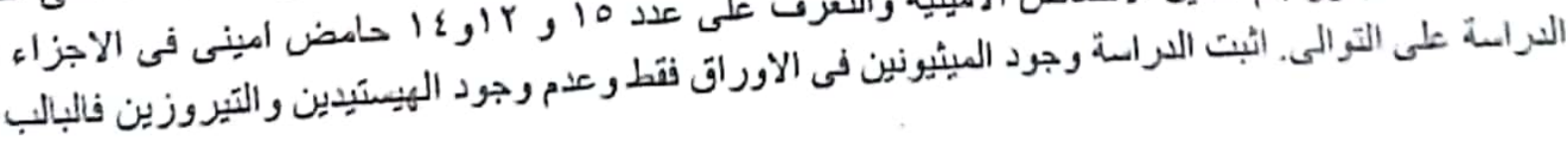

